\title{
SEARCH AND TEST ALGORITHMS FOR TRIPLE PRODUCT PROPERTY TRIPLES
}

\author{
IVO HEDTKE AND SANDEEP MURTHY
}

\begin{abstract}
In 2003 Cohn and UmAns introduced a group-theoretic approach to fast matrix multiplication. This involves finding large subsets of a group $G$ satisfying the Triple Product Property (TPP) as a means to bound the exponent $\omega$ of matrix multiplication.

We present two new characterizations of the TPP, which are useful for theoretical considerations and for TPP test algorithms. With this we describe all known TPP tests and implement them in GAP algorithms. We also compare their runtime. Furthermore we show that the search for subgroup TPP triples of nontrivial size in a nonabelian group can be restricted to the set of all nonnormal subgroups of that group. Finally we describe brute-force search algorithms for maximal subgroup and subset TPP triples. In addition we present the results of the subset brute-force search for all groups of order less than 25 and selected results of the subgroup brute-force search for 2-groups, $\mathrm{SL}_{n} \mathbb{F}_{q}$ and $\mathrm{PSL}_{2} \mathbb{F}_{q}$.
\end{abstract}

\section{INTRODUCTION}

1.1. A Short History of Fast Matrix Multiplication. The naive algorithm for matrix multiplication is an $\mathcal{O}\left(n^{3}\right)$ algorithm. From VOLKER STRASSEN (see Strassen 1969) we know that there is an $\mathcal{O}\left(n^{2.81}\right)$ algorithm for this problem. WinOGRAD optimized STRASSEN's algorithm. While the STRASSEN-WINOGRAD algorithm is the variant that is always implemented (for example in the famous GEMMW package), there are faster ones (in theory) that are impractical to implement. The fastest known algorithm runs in $\mathcal{O}\left(n^{2.38}\right)$ time (see Coppersmith et al. 1987 from Coppersmith and WinOgRAD). Most researchers believe that an optimal algorithm with $\mathcal{O}\left(n^{2}\right)$ runtime exists, but since 1987 no further progress was made in finding one. Because modern microprocessor architectures have complex memory hierarchies and increasing parallelism, performance has become a complex tradeoff, not just a simple matter of counting flops. Algorithms which make use of this technology were described in D'Alberto et al. 2009. Another well known method is Tiling: The normal algorithm can be speeded up by a factor of two by using a six loop implementation that blocks submatrices so that the data passes through the L1 Cache only once.

1.2. The Exponent of the Matrix Multiplication. Let $M(n)$ denote the number of field operations in characteristic 0 required to multiply two $(n \times n)$ matrices. The exponent $\omega$ of the matrix multiplication is defined as

$$
\omega:=\inf \left\{r \in \mathbb{R}: M(n)=\mathcal{O}\left(n^{r}\right)\right\} .
$$

Details about the complexity of matrix multiplication and the exponent $\omega$ can be found in Bürgisser et. al 1997. The result from COPPERSMith and Winograd says that $\omega<2.38$.

1.3. The Group-theoretic Approach from Cohn and Umans. (The reader can find the necessary background on group- and representation theory in Alperin et al. 1991, James et al. 2011. and [Neumann 1994]. More details about the framework can be found in Cohn et al. 2003] and Cohn et al. 2005.)

In 2003 CoHN and Umans introduced in Cohn et al. 2003] a group-theoretic approach to fast matrix multiplication. The main idea is to embed the matrix multiplication over a ring $R$ into the group ring $R G$, where $G$ is a (finite) group. A group $G$ admits such an embedding, if there are subsets $S, T$ and $U$ which fulfill the so-called Triple Product Property.

2000 Mathematics Subject Classification. Primary: 20-04, 68Q25, Secondary: 20D60, 68Q17, 68R05.

The first author was supported by the Studienstiftung des Deutschen Volkes. 
Definition 1.1 (right quotient). Let $G$ be a group and $\emptyset \neq X \subseteq G$ be a nonempty subset of $G$. The right quotient $Q(X)$ of $X$ is defined by $Q(X):=\left\{x y^{-1}: x, y \in X\right\}$.

Note that $Q(S)=S$ holds, if $S$ is a subgroup of $G$.

Definition 1.2 (Triple Product Property). We say that the nonempty subsets $S, T$ and $U$ of a group $G$ fulfill the Triple Product Property (TPP) if for $s \in Q(S), t \in Q(T)$ and $u \in Q(U)$, $s t u=1$ holds iff $s=t=u=1$.

With $\langle n, p, m\rangle$ we denote the problem

$$
\langle n, p, m\rangle: \mathbb{C}^{n \times p} \times \mathbb{C}^{p \times m} \rightarrow \mathbb{C}^{n \times m}, \quad(A, B) \mapsto A B,
$$

of multiplying an $(n \times p)$ with a $(p \times m)$ matrix over $\mathbb{C}$. We say that a group $G$ realizes $\left\langle s_{1}, s_{2}, s_{3}\right\rangle$ if there are subsets $S_{i} \subseteq G$ of sizes $\left|S_{i}\right|=s_{i}$, which fulfill the TPP. In this case we call $\left(S_{1}, S_{2}, S_{3}\right)$ a TPP triple of $G$. Let us now focus on the embedding of the matrix multiplication into $\mathbb{C} G$. Let $G$ realize $\langle n, p, m\rangle$ through the subsets $|S|=n,|T|=p$ and $|U|=m$. Let $A$ be an $(n \times p)$ and $B$ be a $(p \times m)$ matrix. We index the entries of $A$ and $B$ with the elements of $S, T$ and $U$ instead of numbers. Now we have

$$
(A B)_{s, u}=\sum_{t \in T} A_{s, t} B_{t, u} .
$$

CoHn and Umans showed that this is the same as the coefficient of $s^{-1} u$ in the product

$$
\left(\sum_{s \in S, t \in T} A_{s, t} s^{-1} t\right)\left(\sum_{\hat{t} \in T, u \in U} B_{\hat{t}, u} \hat{t}^{-1} u\right) .
$$

So we can read off the matrix product from the group ring product by looking at the coefficients of $s^{-1} u$ with $s \in S$ and $u \in U$.

Definition 1.3 (TPP (subgroup) capacity). We define the TPP capacity $\beta(G)$ of a nontrivial group $G$ as $\beta(G):=\max \{n p m: G$ realizes $\langle n, p, m\rangle\}$ and the TPP subgroup capacity of $G$ as $\beta_{\mathrm{g}}(G):=\max \{n p m: G$ realizes $\langle n, p, m\rangle$ through subgroups $\}$.

Note that $\beta(G) \geq|G|$, because every group $G$ realizes $\langle|G|, 1,1\rangle$ through the TPP triple $(G, 1,1)$.

Definition 1.4 ( $r$-character capacity). Let $G$ be a group with the character degrees $\left\{d_{i}\right\}$. We define the $r$-character capacity of $G$ as $D_{r}(G):=\sum_{i} d_{i}^{r}$.

We can now use $\beta$ and $D_{r}$ to get new bounds for $\omega$ :

Theorem 1.5. Cohn et al. 2003, Thm. 4.1] If $G \neq 1$ is a finite group, then $\beta(G)^{\omega / 3} \leq D_{\omega}(G)$.

Note that this leads to a nontrivial upper bound for $\omega$, iff $\beta(G)>D_{3}(G)$. Therefore, for a fixed group $G$ we search for TPP triples $(S, T, U)$ which maximize $|S| \cdot|T| \cdot|U|$, for example with a brute-force computer search. Note that the maximal $|S| \cdot|T| \cdot|U|$ equals to $\beta(G)$.

With their group theoretic framework COHN and UMANS were able to construct an algorithm for matrix multiplication with running time $\mathcal{O}\left(n^{2.41}\right)$, see Cohn et al. 2005, Sec. 6.3].

From Cohn and Umans we know ([Cohn et al. 2003, Lem. 3.1.]), that $\beta(G)=|G|$ if $G$ is an abelian group. Therefore we only focus on nonabelian groups. We also only focus on finite groups.

1.4. The Aim of this Work. In this article we present two new characterizations of the TPP, which are useful for theoretical considerations and for TPP test algorithms. With this we describe all known TPP tests and implement them in GAP. We also compare their runtime. Furthermore we show that the search for subgroup TPP triples of nontrivial size in a nonabelian group can be restricted to the set of all nonnormal subgroups of that group. Finally we describe bruteforce search algorithms for maximal subgroup and subset TPP triples. In addition we present the results of the subset brute-force search for all groups of order less than 25 and selected results of the subgroup brute-force search for 2-groups, $\mathrm{SL}_{n} \mathbb{F}_{q}$ and $\mathrm{PSL}_{2} \mathbb{F}_{q}$. 


\section{BASIC CONCEPTS}

In this section we present facts that we use in our search algorithms. Details about the subset and subgroup search can be found in section 6. Depending on the context, the symbol 1 will denote either the number 1 , the group identity $1_{G}$, or the trivial subgroup $\left\{1_{G}\right\}$.

Lemma 2.1. Hedtke 2011, Lem. 2 and 3 and Thm. 4]

$\left(^{*}\right)$ Let $\emptyset \neq X \subseteq G$ be a nonempty subset of a group $G$ and $g \in G$. Then $1 \in Q(X)$ and $g \in Q(X) \Leftrightarrow g^{-1} \in Q(X)$.

(**) If $S, T$ and $U$ fulfill the TPP then $Q(X) \cap Q(Y)=1$ holds for all $X \neq Y \in\{S, T, U\}$.

(***) If $(S, T, U)$ is a TPP triple with $1 \in S \cap T \cap U$, then $S \cap T=T \cap U=S \cap U=1$.

Lemma 2.2. [Hedtke 2011, Cor. 6] If $(S, T, U)$ is a TPP triple of $G$, then $|S|+|T|+|U| \leq|G|+2$.

First we note, that we are only interested in matrix-matrix multiplication, that means, we consider only TPP triples $(S, T, U)$ with $|S|,|T|,|U|>1$. Furthermore we have: Assume that in a TPP triple of $G$ one of $S, T$ or $U$ is $G$ itself. Then it follows from the lemma above, that the other two sets of the triple have size 1 . Because we omit this case, we only use:

Observation 2.3. It is sufficient to search TPP triples with $|S|,|T|,|U| \in\{2, \ldots,|G|-1\}$.

From Cohn and Umans (see Cohn et al. 2003, Lem. 2.1]) we know the following symmetry property:

Lemma 2.4. If $G$ realizes $\langle n, p, m\rangle$, then it does so for every permutation of $n, p$ and $m$.

We conclude the following:

Observation 2.5. It is sufficient to search TPP triples with $|S| \geq|T| \geq|U|$.

We know, that $\beta(G) \geq|G|$, therefore we are only interested in

Observation 2.6. It is sufficient to search TPP triples with $|S| \cdot|T| \cdot|U|>|G|$.

Now we can combine the last two observations: We know that $|S| \cdot|T| \cdot|U| \leq|S|^{3}$. Therefore we have

Observation 2.7. It is sufficient to search TPP triples with $|S| \geq\lceil\sqrt[3]{|G|}\rceil=: \ell(G)$, where $\lceil x\rceil$ denotes the smallest integer not smaller than $x$.

Assume the case, where we try to find triples that yield a nontrivial upper bound for $\omega$ instead of only finding nontrivial TPP triples. In this case we change the $|G|$ in the last two observations to $D_{3}(G)$.

Definition 2.8 (basic TPP triple). According to Neumann we call a TPP triple $(S, T, U)$ that fulfills $1 \in S \cap T \cap U$ a basic TPP triple.

From Neumann we know the following facts that reduce the search space enormously:

Lemma 2.9. Neumann 2011, Obs. 2.1] If $(S, T, U)$ is a TPP triple of $G$, then $(d S a, d T b, d U c)$ is a TPP triple for all $a, b, c, d \in G$, too.

Note that any TPP triple can be translated to a basic TPP triple by lemma 2.9 . Together with $(* * *)$ it follows, that:

Observation 2.10. It is sufficient to search TPP triples with $S \cap T=T \cap U=S \cap U=1$.

Lemma 2.11. Neumann 2011, Obs. 3.1] If $(S, T, U)$ is a TPP triple, then $|S|(|T|+|U|-1) \leq|G|$, $|T|(|S|+|U|-1) \leq G$ and $|U|(|S|+|T|-1) \leq|G|$.

Observation 2.12. It is sufficient to search TPP triples with $|S|(|T|+|U|-1) \leq|G|$.

Example 2.13. Let us focus on a brute-force search for TPP triples of subgroups in the MATHIEU group $M_{11}$. We pick out this example because it is a big group, note that $\left|M_{11}\right|=7920$, with many (exactly 8651) subgroups. Possible orders for $S, T$ or $U$ are (computed with GAP):

$\{1,2,3,4,5,6,8,9,10,11,12,16,18,20,24,36,48,55,60,72,120,144,360,660,720,7920\}=: \mathcal{P}$. 
IVO HEDTKE AND SANDEEP MURTHY

Note that $\mathcal{P}$ has 26 elements. If we use observation 2.3, we define $\mathcal{P}:=\mathcal{P} \backslash\{1,7920\}$, so we have $|\mathcal{P}|=24$. A naive idea would be $(|S|,|T|,|U|) \in \mathcal{P}^{3}=: C$. Because $|C|=13824$ we would have a large search space for a brute-force search. Now we use observation 2.5 and achieve $|C|=2600$. If we only search for subgroup TPP triples that yield a nontrivial bound for $\omega$, we use observation 2.6 with $D_{3}(G)=355208$ (again computed with GAP) instead of $|G|$. With this it follows that $|C|=404$. Finally we use NEumAnns inequality from observation 2.12, which yields to $|C|=0$. This example shows that the observations above reduce the search space for a brute-force search enormously. In this case it is not even necessary to start a search.

\section{Two New Characterizations of the Triple Product Property}

In this section we present two new characterizations of the TPP. They are useful for theoretical considerations (in particular theorem 3.1 and for TPP test algorithms which we discuss in section 5 .

Theorem 3.1. Three subsets of $G$ form a basic TPP triple $(S, T, U)$ iff
(i) $1 \in S \cap T \cap U$,
(ii) $Q(T) \cap Q(U)=1 \quad$ and
(iii) $Q(S) \cap Q(T) Q(U)=1$.

This is not a limitation, because we only need to search for basic TPP triples.

Proof. First assume that $(S, T, U)$ is a basic TPP triple. (i) follows directly from the definition and (ii) from (**). Furthermore (*) implies that $1 \in Q(S) \cap Q(T) Q(U)$. Now assume there is a common element $1 \neq x \in Q(S) \cap Q(T) Q(U)$. Then $x=s=t u$, for some $s \in Q(S)$ and $t u \in Q(T) Q(U)$. This means $1=x^{-1} x=s^{-1} t u$, but the TPP for $(S, T, U)$ implies that $1=s^{-1}=t=u$ and therefore $x=1$, a contradiction.

Now assume that the equations (i)-(iii) hold for the subsets $S, T$ and $U$. Consider the triple quotient product $s t u$ for arbitrary elements $s \in Q(S), t \in Q(T)$ and $u \in Q(U)$. Then stu=1 is equivalent to $s^{-1}=t u$. Now (iii) implies that $s^{-1}=t u=1$ and (*) together with (ii) imply that $t=u=1$ and so $(S, T, U)$ is a basic TPP triple.

Definition 3.2 (subtransversal, support). Let $C$ be a finite nonempty set and $\mathcal{C}=\left\{C_{1}, \ldots, C_{k}\right\}$ a partition of it. A set $X \subseteq C$ is called a subtransversal for $\mathcal{C}$ with support $\operatorname{supp}_{\mathcal{C}}(X)=\mathcal{T} \subseteq \mathcal{C}$ if for all $C_{i} \in \mathcal{C}$

$$
\left|X \cap C_{i}\right|= \begin{cases}1 & C_{i} \in \mathcal{T} \\ 0 & \text { otherwise }\end{cases}
$$

It then follows that $|X|=|\mathcal{T}|$. In the special case when the collection $\mathcal{C}$ is the set of left (or right) cosets of a subgroup $S$ of a group $G$, then any subtransversal $T$ for $G / S$ (or $S \backslash G$ ) will simply be called a subtransversal for $S$ in $G$.

Theorem 3.3. Let $G$ be a group, $S$ a subgroup of $G$, and $T, U$ subsets of $G$.

(1) If $(S, T, U)$ is a basic TPP triple of $G$ then $T$ and $U$ are subtransversals for $S$ in $G$ such that

$$
\operatorname{supp}_{S \backslash G}(T) \cap \operatorname{supp}_{S \backslash G}(U)=\{S\} .
$$

(2) If $T$ and $U$ are also subgroups of $G$, and $T$ and $U$ are subtransversals for $S$ in $G$ satisfying (1) then $(S, T, U)$ is a TPP triple of $G$.

Proof. (i) We have $S \cap T=S \cap U=1$, by (***). Let $S \neq S r \in S \backslash G$ be a nontrivial coset of $S$. Assume distinct elements $1 \neq t, t^{\prime} \in T \cap S r$, where $t=s r$ and $t^{\prime}=s^{\prime} r$ for distinct $s, s^{\prime} \in S$. Then $1 \neq t^{\prime} t^{-1}=s^{\prime} s^{-1} \in S \cap Q(T)$, and that contradicts the TPP requirement (**). So for any coset $S r \in S \backslash G$, we have $|T \cap S r| \leq 1$. The same holds for $U$. Therefore $T$ and $U$ are subtransversals for $S \backslash G$. Now assume distinct elements $1 \neq t=s r \in T \cap S r$ and $1 \neq u=s^{\prime} r \in U \cap S r$ for a nontrivial coset $S r \neq S$. Then $1 \neq t u^{-1}=s\left(s^{\prime}\right)^{-1} \in Q(S) \cap Q(T) Q(U)=S \cap Q(T) Q(U)$ and that contradicts theorem 3.1. This shows that $T$ and $U$ fulfill (1).

(ii) Assume $T$ and $U$ are subtransversals of $S \backslash G$ and fulfill (1). Since $S, T$ and $U$ are subgroups we have $1 \in S \cap T \cap U$ and $1 \in S \cap T U$. Because the intersection of the supports of $T$ and $U$ is $\{S\}, T \cap U=1$ holds. Now assume that there is an $1 \neq x \in S \cap T U$. So there are $s \in S, t \in T$ 
and $u \in U$ with $1 \neq s=t u$. Therefore we have $t u \in S$, which is equivalent to $t \in S u^{-1}$. But then would be $t=u=1$, a contradiction. So $(S, T, U)$ is a TPP triple by theorem 3.1 .

Observation 3.4. Three subgroups $S, T, U$ of a group $G$ form a TPP triple iff $T$ and $U$ are subtransversals for $S$ in $G$, satisfying (1).

Theorem 3.5. Let $G$ be a group. If $(S, T, U)$ is a TPP triple of subgroups where at least one of $S$, $T$ or $U$ is normal in $G$, then $|S| \cdot|T| \cdot|U| \leq|G|$.

Proof. Without loss of generality assume that $S$ is nontrivial, proper and normal in $G$. Because $S$ is normal in $G$, we have $S \backslash G=G / S$. We will work with $G / S$. From theorem 3.3 we know, that $T$ and $U$ are subtransversals for $G / S$ that fulfill $(1)$. Let $S_{1}:=S, S_{2}, \ldots, S_{v}$ be the $v=[G: S] \geq 2$ elements of $G / S$. We define $\mathcal{T}:=\operatorname{supp}_{G / S}(T) \subseteq G / S$ and $\mathcal{U}$ in the same way. Then $\mathcal{T}$ and $\mathcal{U}$ are subgroups of $G / S$ with $|\mathcal{T}|=|T|,|\mathcal{U}|=|U|$ and $\mathcal{T} \cap \mathcal{U}=1$. To see this, first consider $\mathcal{T}$. Because $T$ and $U$ are subtransversals for $G / S$ that fulfill $(1)$, we have $1_{G / S}=S \in \mathcal{T}$. Now let $r S, r^{\prime} S \in \mathcal{T}$ be cosets of $S$. So there are $t, t^{\prime} \in T$ such that $T \cap r S=\{t=r s\}$ and $T \cap r^{\prime} S=\left\{t^{\prime}=r^{\prime} s^{\prime}\right\}$, where $s, s^{\prime} \in S$. They have the product $r s r^{\prime} s^{\prime}=t t^{\prime} \in T \cap r r^{\prime} S$ and so $\left|T \cap r r^{\prime} S\right| \geq 1$. Since $T$ is a subtransversal for $G / S$ it follows that $\left|T \cap r r^{\prime} S\right|=1$ and so $r r^{\prime} S \in \mathcal{T}$. The inverse of any given $r S \in \mathcal{T}$ is $r^{-1} S \in G / S$ and if $t \in T$ is such that $T \cap r S=\{t\}$ then $t=r s$ for some $s \in S$, and $t^{-1}=s^{-1} r^{-1} \in S r^{-1}=r^{-1} S$ (because $S$ is normal), and so $t^{-1} \in T \cap r^{-1} S$, and we can conclude that $r^{-1} S \in \mathcal{T}$ as well. The same holds for $\mathcal{U}$. Because (1) we know that $\mathcal{T} \cap \mathcal{U}=1$ and so $(\mathcal{T}, \mathcal{U}, 1)$ is a TPP triple of $G / S$ by theorem 3.1. From Neumanns inequality (see lemma 2.9 it follows that $|\mathcal{T}|(|\mathcal{U}|+1-1)=|\mathcal{T}| \cdot|\mathcal{U}|=|T| \cdot|U| \leq|G / S|=|G| /|S|$, which we wanted to show.

We can use the result above to create an additional filter for the search space of subgroup TPP triples:

Observation 3.6. If we are only interested in subgroup TPP triples of nontrivial size it is suffcient to search for $S, T$ and $U$ that are all nonnormal.

\section{On the TPP Capacity of Nonabelian Groups}

In this section we present some facts about the TPP capacity of nonabelian groups. The following two results state nontrivial lower bounds for $\beta(G)$. The ideas behind the proofs are those of the authors, but the proofs as formulated here are those of NEUMANN.

Lemma 4.1. If $G$ is a nonabelian group with a nonnormal subgroup $S$ of index $[G: S]=3$, then $\beta(G) \geq \frac{4}{3}|G|$.

Proof. Let $S \backslash G$ be the right coset space of $G$ of size $[G: S]=3$. There is a natural homomorphism $\phi: G \rightarrow S_{3}$, defined by $g \mapsto \pi_{g}$ for all $g \in G$, where $\pi_{g}$ describes a permutation action $(g, S r) \mapsto$ $S r g=S r_{g} \in S \backslash G$ for all $S r \in S \backslash G$, of $g$ on $S \backslash G$. The homomorphism $\phi$ has a kernel $K:=\operatorname{ker} \phi \unlhd G$, which is $\operatorname{Core}_{G}(S)$ (the largest normal subgroup of $G$ contained in $S$ ). The quotient group $G / K \cong \operatorname{img} \phi \leq S_{3}$ is isomorphic to a transitive subgroup in $S_{3}$. The nontrivial transitive subgroups of $S_{3}$ are $A_{3}$ and $S_{3}$ itself. Since $S$ is nonnormal, $K<S$ is proper and so $|G / K|>3$. Thus $G / K \cong S_{3}$. From Cohn et al. 2003. Lem. 2.2] we know that $\beta(G) \geq \beta(G / K) \beta(K)$. It follows (see table 1), that $\beta(G) \geq 8 \beta(K) \geq 8|K|=8|G| /|G / K|=\frac{8}{6}|G|=\frac{4}{3}|G|$.

Lemma 4.2. If $G$ is a nonabelian group with a self-normalising subgroup $S$ of index $[G: S]=4$, then $\beta(G) \geq \frac{3}{2}|G|$.

Proof. The subgroup $S$ has a normal core $K:=\operatorname{Core}_{G}(S) \unlhd G$ which is proper $K<S$, such that the quotient group $G / K$ is of order $|G / K|>|G / S|=4$. The core $K$ is the kernel of the natural homomorphism $\phi: G \rightarrow S_{4}$ which describes the permutation action of $G$ on $G / S$, and so $G / K \cong \operatorname{img} \phi \leq S_{4}$ is a transitive subgroup of order greater than 4 . The only possibilities are $A_{4}$, $D_{8}$ and $S_{4}$. Because we assume that $S$ is self-normalising, the case $G / K \cong D_{8}$ is not feasible. The statement follows from $\beta\left(A_{4}\right) /\left|A_{4}\right|=\beta\left(S_{4}\right) /\left|S_{4}\right|=3 / 2$ (see table 1 like in the proof of lemma 4.1. 


\section{Triple Product Property Test Algorithms}

At the moment there are five algorithms to test the TPP. In this section we will present them and compare their running time.

We start with two algorithms that directly came from the TPP definition. We define $L:=$ $Q(S) Q(T) Q(U)$ as a list, not as a set. Then we count, how many 1's are in it. The TPP is fulfilled iff there is only one 1 in $L$.

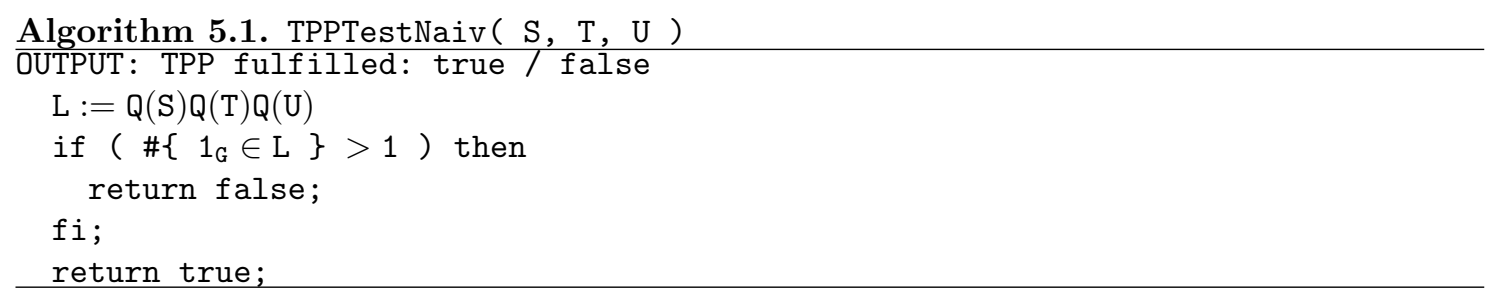

The algorithm above is very naive, because we use the complete list $L$. This needs a lot of time and memory. It is a better idea to search element-wise for 1's.

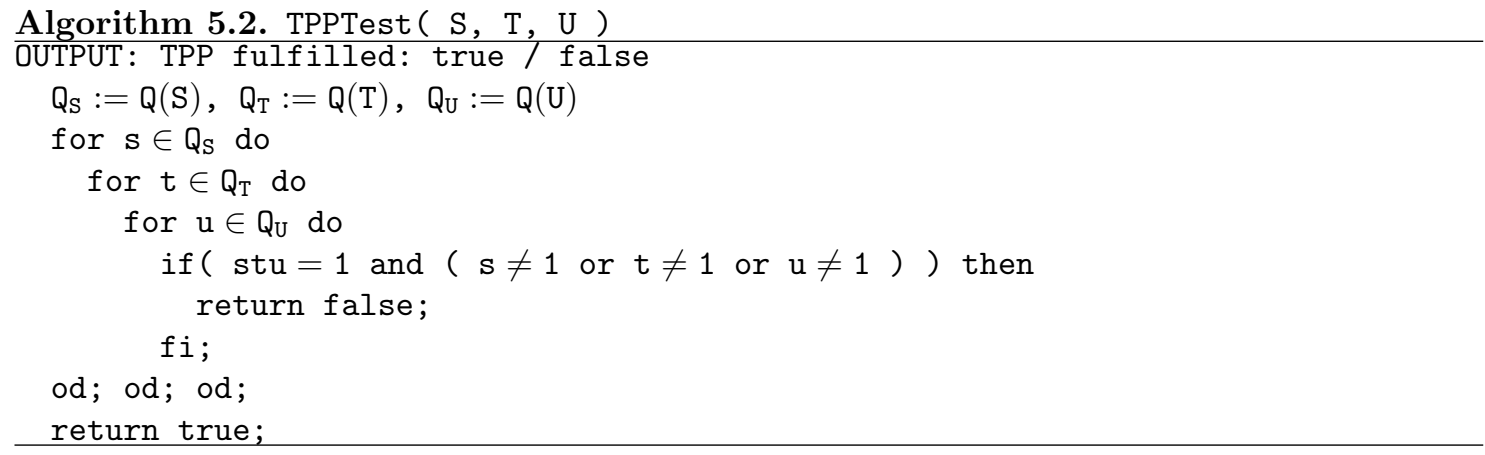

From HENDRIK OREM we know the following equivalent form of the TPP:

Lemma 5.3. Orem 2009, Thm. 2.1] Subsets $S, T$ and $U$ of $G$ satisfy the TPP iff

$$
\left|S^{-1}\right| \cdot|U|=\left|S^{-1} U\right| \quad \text { and } \quad\left(S^{-1}(Q(T) \backslash 1) U\right) \cap S^{-1} U=\emptyset .
$$

Note that for a TPP test based on the lemma above we only need to compute one of the right quotients, instead of all three right quotients in the original TPP definition.

Algorithm 5.4. TPPTestOrem( $\mathrm{S}, \mathrm{T}, \mathrm{U})$

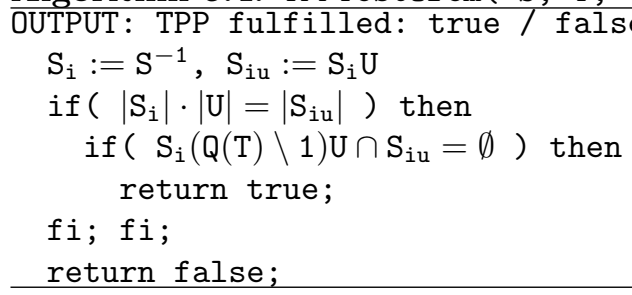

The fourth algorithm comes from the TPP reformulation of theorem 3.1 it requires a basic triple $(1 \in S \cap T \cap U)$ as input:

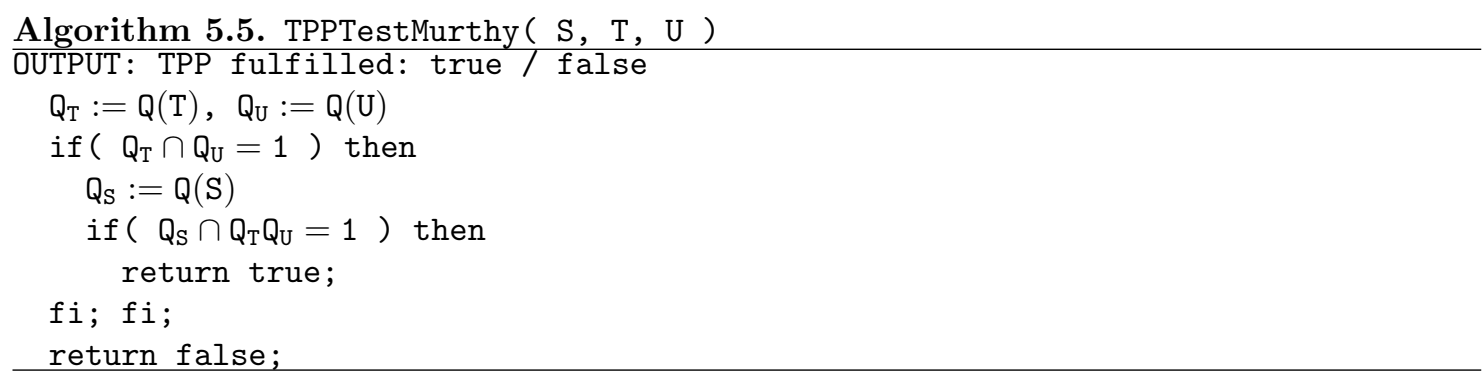


Now we focus on TPP tests for subgroups. We start with the TPP test inspired by theorem 3.3 about subtransversals.

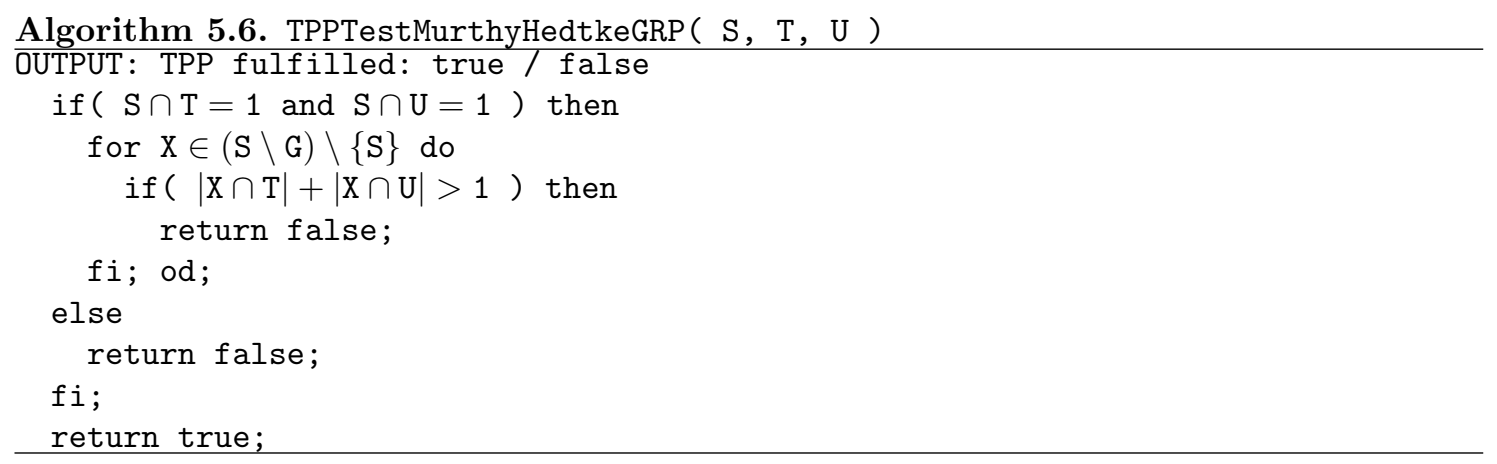

In the case where $S, T$ and $U$ are subgroups, we have $Q(S)=S, Q(T)=T$ and $Q(U)=U$. Therefore the remaining test algorithms for subgroups are:
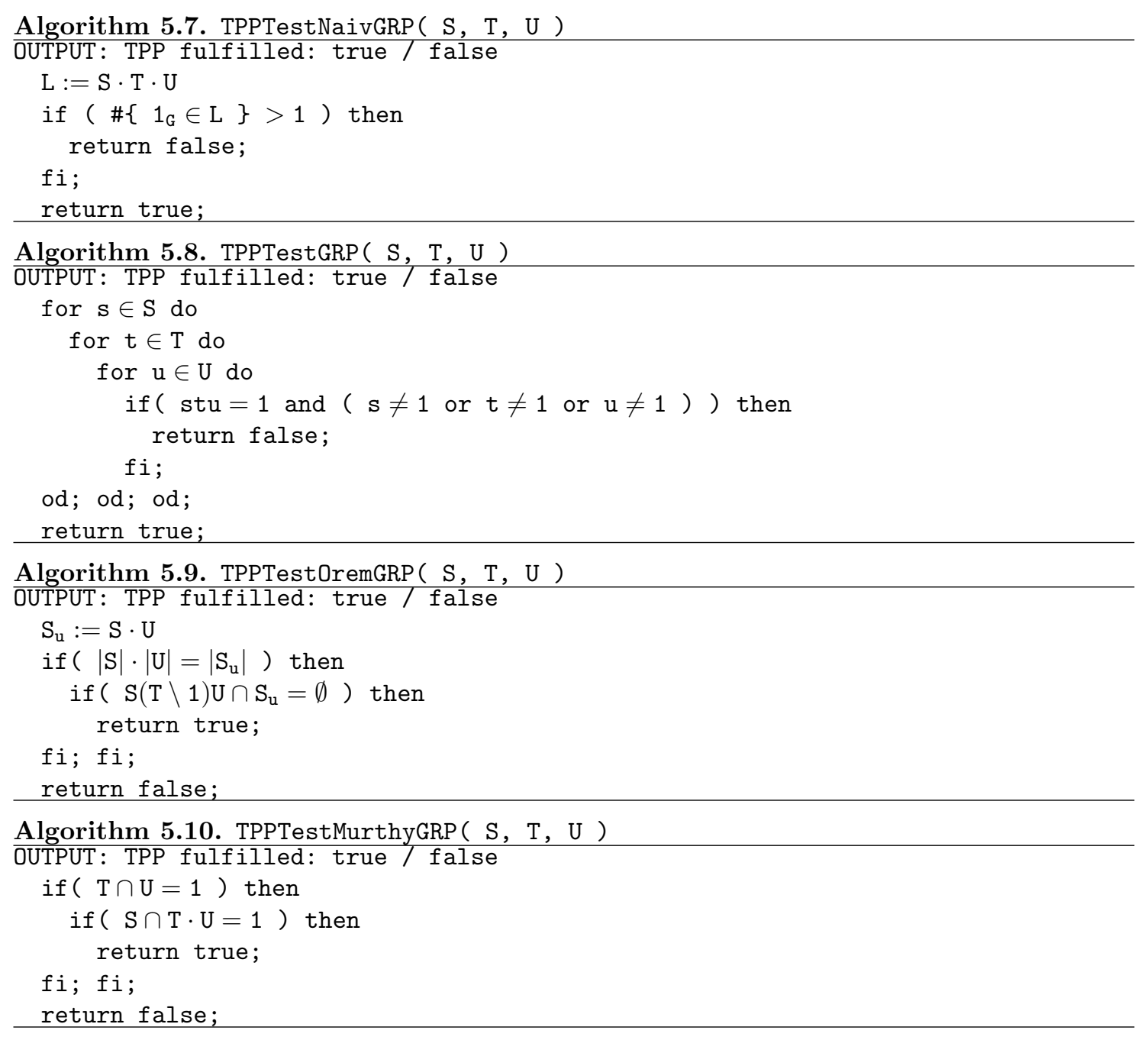

Now we compare the running time of the different TPP tests. For this we use the search algorithms described in the next section with the different test algorithms. We use a GAP (GAP 2008) implementation of the algorithms in this paper with the SONATA package ( Aichinger et. al 2008) for the Subgroups routine. The interested reader can get the GAP codes from the first author via email. 


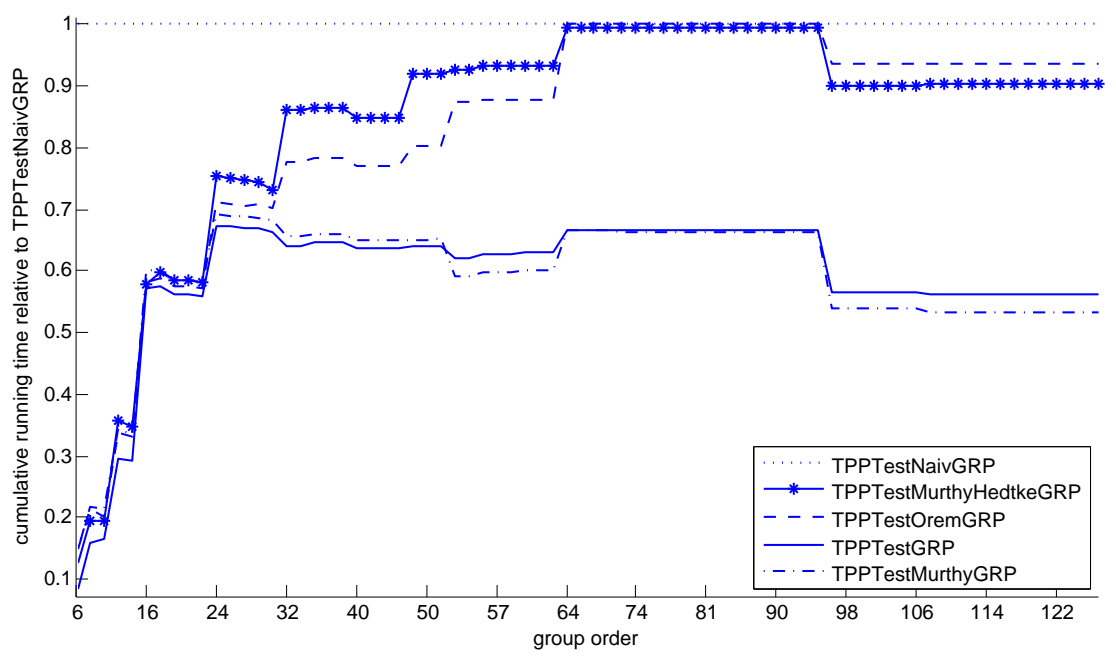

FIGURE 1. Cumulative running time relative to TPPTestNaivGRP of the bruteforce search for subgroups with the five different test algorithms. (The vertical axis is measuring the cumulative runtime of a sequence of 10 runs for each of the five different TPP verification algorithms, averaged by 10, so the closer the lines are to the zero line, the better.)

For the subgroup test algorithms we run the brute-force search algorithm 10 times (except for the groups of order 64 and 96, because there are more than 200 such groups) for each nonabelian group of order less than 128 . The results in figure 1 show the mean cumulative running time of the search algorithm for all nonabelian groups up to a given order. We recommend to use TPPTestMurthyGRP.

For the subset test algorithms we do the same, but only up to group order 20 and only with 2 repeats to build the mean value. The results are shown in figure 2 (left). It is very obvious, that compared to the naive TPP test the algorithms 5.2, 5.4 and 5.5 are very fast. Therefore figure 2 (right) shows the details. Again, we recommend to use TPPTestMurthy.
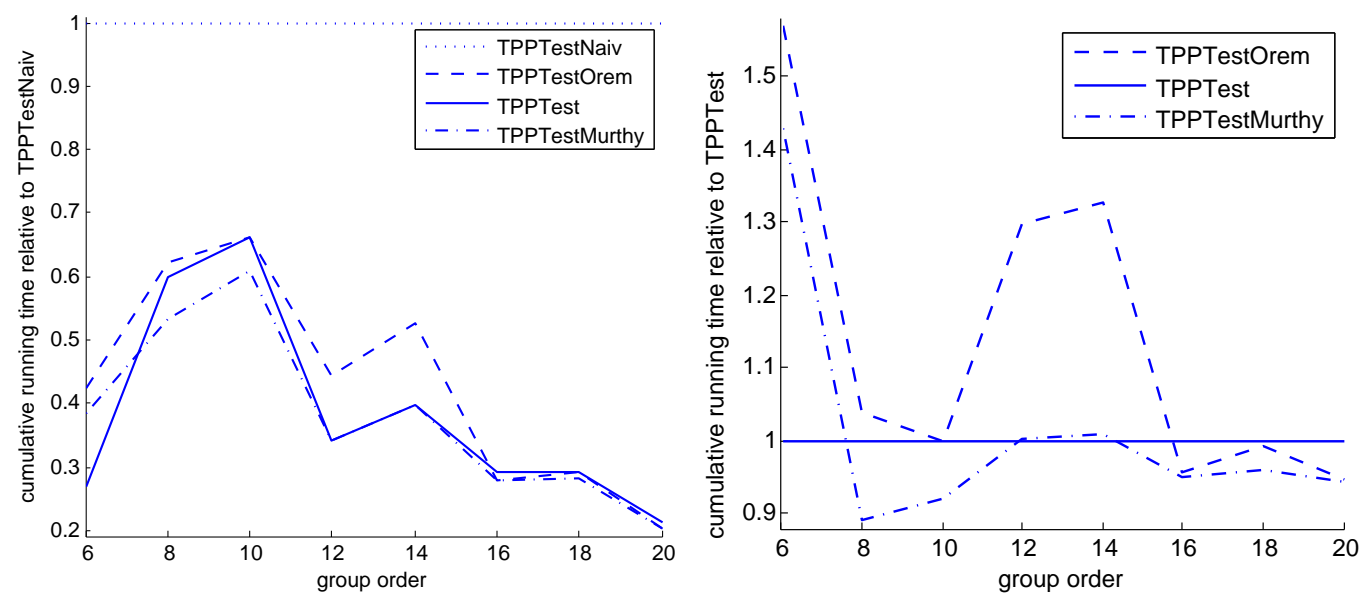

Figure 2. Left: Cumulative running time relative to TPPTestNaiv of the bruteforce search for subsets with the four different test algorithms. Right: Cumulative running time of TPPTestOrem and TPPTestMurthy relative to TPPTest. 


\section{Brute-Force SEArCh}

In this section we describe the brute-force search algorithms that we use for our computations. We use the observations from section 2 to reduce the search space for $S, T, U \subseteq G$ and the TPP tests from the last section. Although it is relatively quick to search for subgroup TPP triples, they do not fully describe the TPP capacity of a group, as the following theorem shows.

Theorem 6.1. For every finite group $G, \beta(G) \geq \beta_{\mathrm{g}}(G)$ holds. There are groups with $\beta(G)>$ $\beta_{\mathrm{g}}(G)$.

Proof. The first statement is trivial, because the search space for $\beta$ includes the one for $\beta_{\mathrm{g}}$. For the second statement consider the group $D_{10}=\left\langle d, s: s^{2}=d^{5}=1, s d s=d^{-1}\right\rangle$ of order 10. From table 1 we know that $\beta_{\mathrm{g}}\left(D_{10}\right)=10$. But the subsets $S:=\langle s\rangle, T:=\{d, s\}$ and $U:=\left\{1, s d, d^{3}\right\}$ of $D_{10}$ realize the problem $\langle 2,2,3\rangle$ of size 12 , and so $\beta\left(D_{10}\right)=12$.

From the result above we see, that it is necessary so search for subset TPP triples. Therefore we present search algorithms for either subgroup TPP triples and for subset TPP triples.

6.1. Subgroup Search. We use the following algorithm to search for subgroup TPP triples in a given nonabelian group. Note that we use the Subgroups routine from the SONATA package and that the subgroups returned from that command are ordered ascending by the sizes of the subgroups. Our command NonnormalSubgroups is only a filter for the nonnormal Subgroups of $G$.

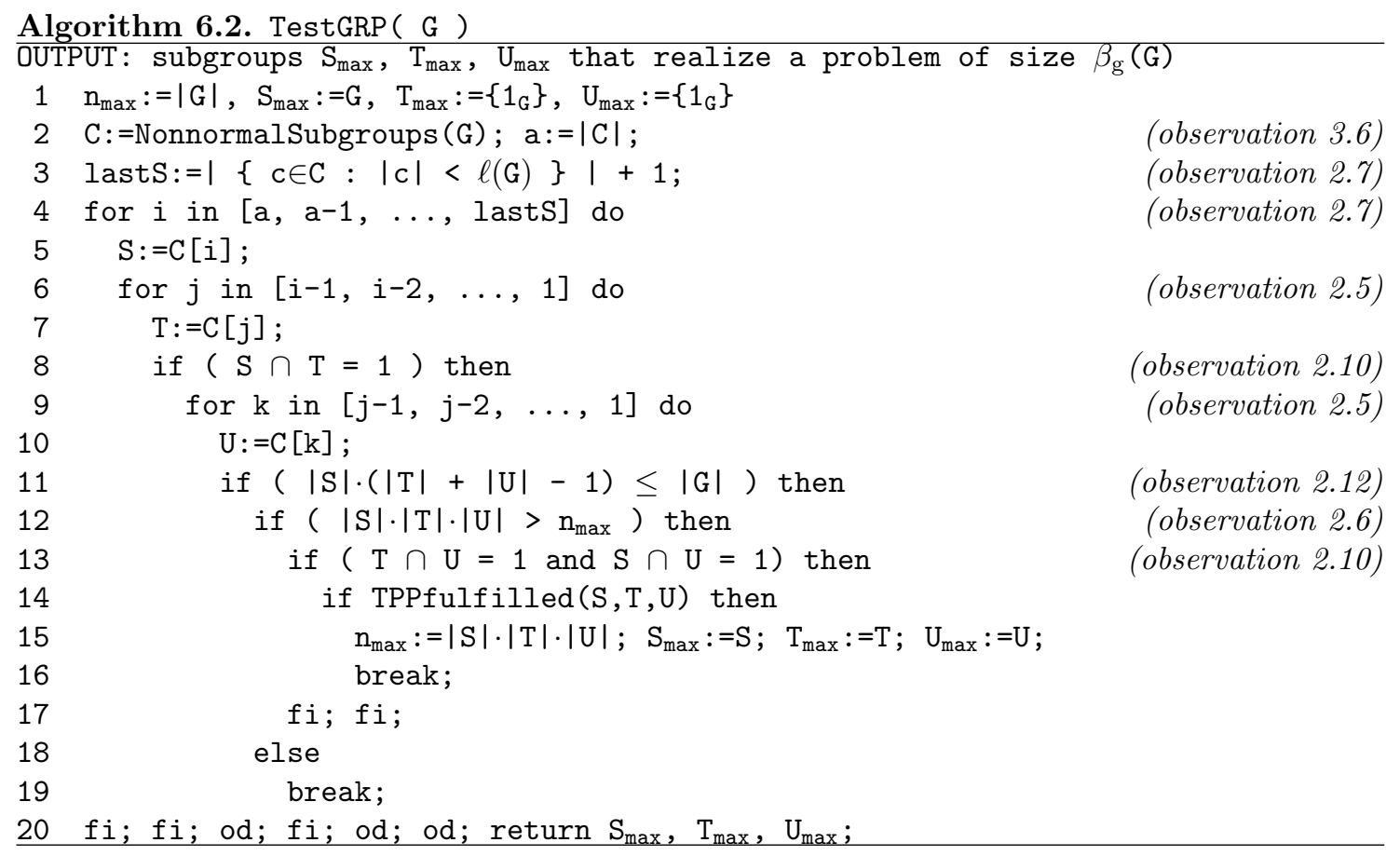

For the command TPPfulfilled in line 14 of the code we use one of the TPP tests for subgroups described in the previous section. The break command in line 16 is used because all other $U$ that occur in the inner for loop have a size smaller or equal than $U_{\max }$ and we don't need to test them, because we will not get a bigger TPP triple. The break command in line 19 is used because the multiplicative size of all other TPP triples with an $U$ from the inner loop have a size smaller than $n_{\max }$.

6.2. Subset Search. The search for subset TPP triples is very similar to the subgroup search. But instead of the Subgroups routine we use a method to generate subsets of $G$. We followed an idea of E. BurnetT (see Burnett 2010]) to generate the subsets on the fly in our code. (We also had the idea to generate a binary representation of the subsets. Each subset of $G$ can be identified 
as an element of $\{0,1\}^{|G|}$. But it needs too much memory to generate the whole object $\{0,1\}^{|G|}$ and therefore we are glad, that we found a way to generate the subsets one after another.)

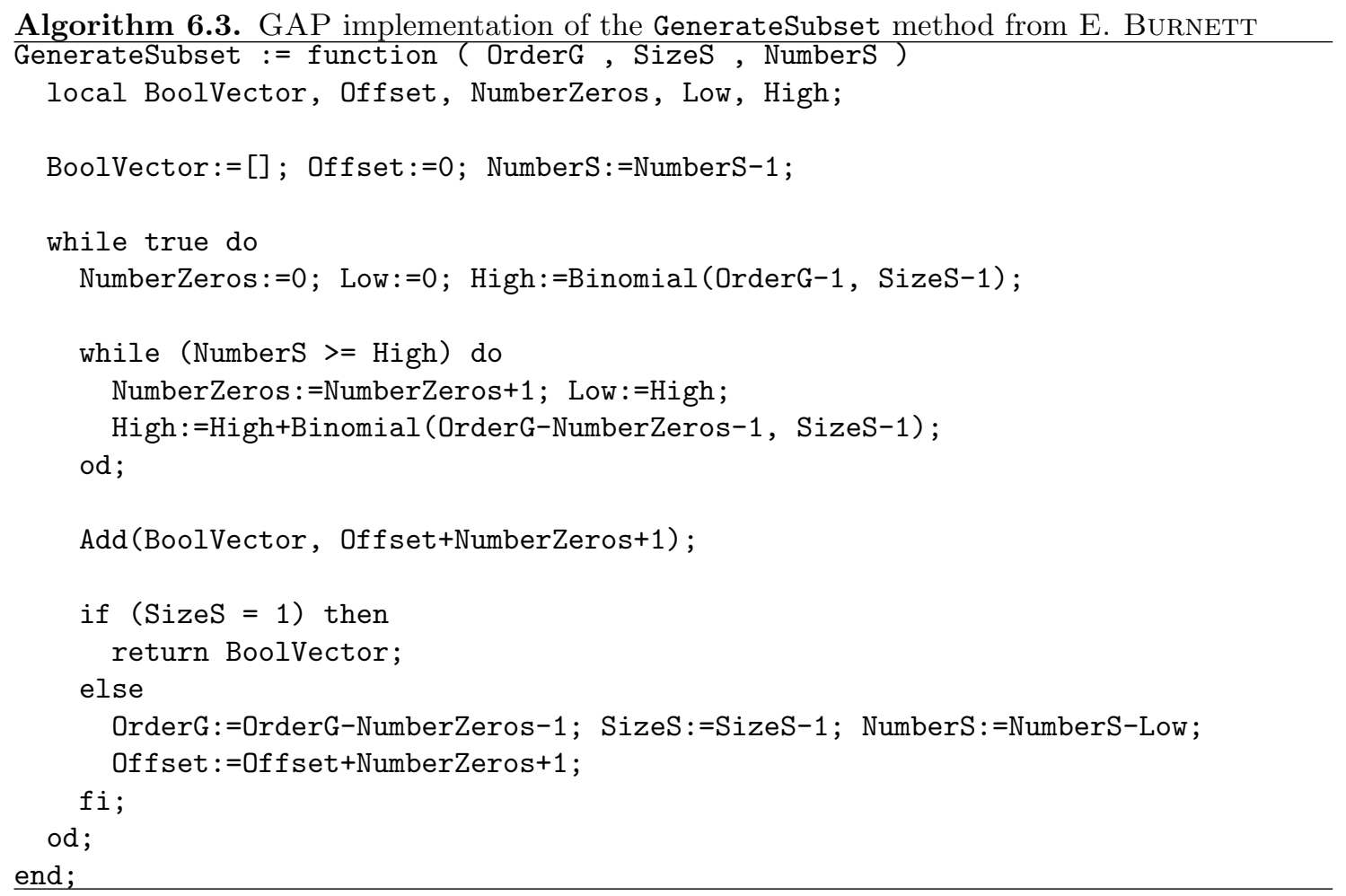

The differences to the subgroup search algorithm are: First we compute the set $W$ of all possible values of $|S|,|T|$ and $|U|$, that means $W:=\{2, \ldots,|G|-1\}^{3}$. Then we use all observations from section 2 to reduce the search space. Now we order the elements of $W$ decreasing by their multiplicative size (we use this to stop the algorithm in the case that we found a TPP triple, because it is the biggest TPP triple of $G$ ). In the outer for loop we go through all $i \in W$ and generate in the inner loops the $\left(\begin{array}{c}|G|-1 \\ i_{1}-1\end{array}\right)$ subsets $S$ that contain the identity of $G$ and the $U$ 's and $T$ 's in the same way.

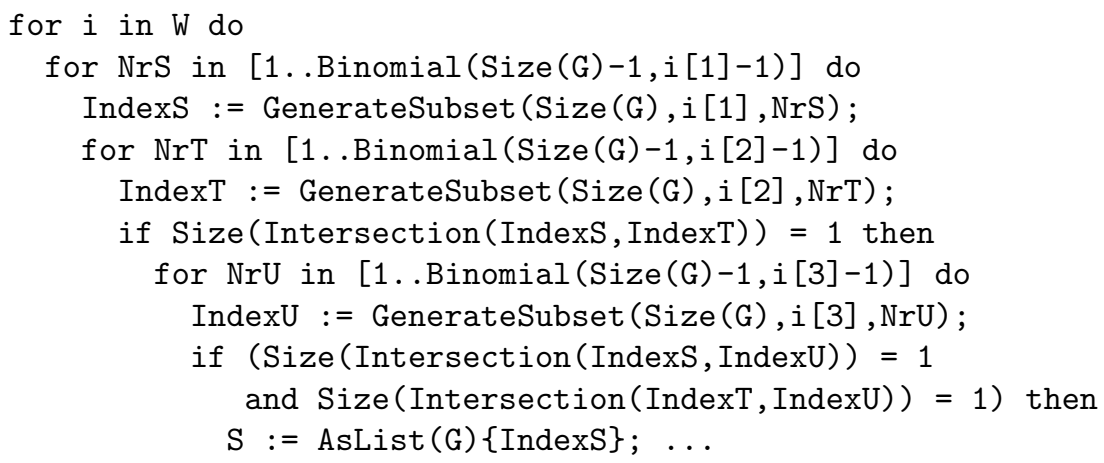

Of course one has to use a TPP test for subsets (see the previous section).

\section{Selected Results}

In this section we present some computational results of the brute-force search with subsets and subgroups. The values in the following tables are:

- GAP Id: The IdSmallGroup in the SmallGroups Library.

- $D_{3}(G)$ resp. $D_{3}$ : The 3 -character capacity (see definition 1.4).

- $\beta(G)$ resp. $\beta$ : The TPP capacity (see definition 1.3). 


\begin{tabular}{|c|c|c|c|c|c|c|c|}
\hline GAP Id & Group & $D_{3}(G)$ & $\beta(G)$ & $\beta_{\mathrm{g}}(G)$ & $\beta / D_{3}$ & $\boldsymbol{\beta} /|\boldsymbol{G}|$ & $\langle n, p, m\rangle$ \\
\hline$[6,1]$ & $\overline{S_{3}}$ & 10 & 8 & 8 & 0.8 & 1.33333 & $2,2,2$ \\
\hline$[8,3]$ & $D_{8}$ & 12 & 8 & 8 & 0.666667 & 1 & $2,2,2$ \\
\hline$[8,4]$ & $Q_{8}$ & 12 & 8 & 8 & 0.666667 & 1 & $2,2,2$ \\
\hline$[10,1]$ & $D_{10}$ & 18 & 12 & 10 & 0.666667 & 1.2 & $3,2,2$ \\
\hline$[12,1]$ & $C_{3} \rtimes C_{4}$ & 20 & 16 & 12 & 0.8 & 1.33333 & $4,2,2$ \\
\hline$[12,3]$ & $A_{4}$ & 30 & 18 & 18 & 0.6 & 1.5 & $3,3,2$ \\
\hline$[12,4]$ & $D_{12}$ & 20 & 16 & 16 & 0.8 & 1.33333 & $4,2,2$ \\
\hline$[14,1]$ & $D_{14}$ & 26 & 16 & 14 & 0.615385 & 1.14286 & $4,2,2$ \\
\hline$[16,3]$ & $\left(C_{4} \times C_{2}\right) \rtimes C_{2}$ & 24 & 16 & 16 & 0.666667 & 1 & $4,2,2$ \\
\hline$[16,4]$ & $C_{4} \rtimes C_{4}$ & 24 & 16 & 16 & 0.666667 & 1 & $4,2,2$ \\
\hline$[16,6]$ & $C_{8} \rtimes C_{2}$ & 24 & 16 & 16 & 0.666667 & 1 & $4,2,2$ \\
\hline$[16,7]$ & $D_{16}$ & 28 & 20 & 16 & 0.714286 & 1.25 & $5,2,2$ \\
\hline$[16,8]$ & $Q D_{16}$ & 28 & 16 & 16 & 0.571429 & 1 & $4,2,2$ \\
\hline$[16,9]$ & $Q_{16}$ & 28 & 16 & 16 & 0.571429 & 1 & $4,2,2$ \\
\hline$[16,11]$ & $C_{2} \times D_{8}$ & 24 & 16 & 16 & 0.666667 & 1 & $4,2,2$ \\
\hline$[16,12]$ & $C_{2} \times Q_{8}$ & 24 & 16 & 16 & 0.666667 & 1 & $4,2,2$ \\
\hline$[16,13]$ & $\left(C_{4} \times C_{2}\right) \rtimes C_{2}$ & 24 & 16 & 16 & 0.666667 & 1 & $4,2,2$ \\
\hline$[18,1]$ & $D_{18}$ & 34 & 24 & 24 & 0.705882 & 1.33333 & $6,2,2$ \\
\hline$[18,3]$ & $C_{3} \times S_{3}$ & 30 & 24 & 24 & 0.8 & 1.33333 & $6,2,2$ \\
\hline$[18,4]$ & $C_{3}^{2} \rtimes C_{2}$ & 34 & 24 & 24 & 0.705882 & 1.33333 & $6,2,2$ \\
\hline$[20,1]$ & $C_{5} \rtimes C_{4}$ & 36 & 24 & 20 & 0.666667 & 1.2 & $6,2,2$ \\
\hline$[20,3]$ & $C_{5} \rtimes C_{4}$ & 68 & 32 & 32 & 0.470588 & 1.6 & $4,4,2$ \\
\hline$[20,4]$ & $D_{20}$ & 36 & 24 & 20 & 0.666667 & 1.2 & $6,2,2$ \\
\hline$[21,1]$ & $C_{7} \rtimes C_{3}$ & 57 & 27 & 27 & 0.473684 & 1.28571 & $3,3,3$ \\
\hline$[22,1]$ & $D_{22}$ & 42 & 28 & 22 & 0.666667 & 1.27273 & $7,2,2$ \\
\hline$[24,1]$ & $C_{3} \rtimes C_{8}$ & 40 & 32 & 24 & 0.8 & 1.33333 & $4,4,2$ \\
\hline$[24,3]$ & $\mathrm{SL}_{2} \mathbb{F}_{3}$ & 54 & 36 & 36 & 0.666667 & 1.5 & $4,3,3$ \\
\hline$[24,4]$ & $C_{3} \rtimes Q_{8}$ & 44 & 32 & 24 & 0.727273 & 1.33333 & $4,4,2$ \\
\hline$[24,5]$ & $C_{4} \times S_{3}$ & 40 & 32 & 32 & 0.8 & 1.33333 & $4,4,2$ \\
\hline$[24,6]$ & $D_{24}$ & 44 & 32 & 32 & 0.727273 & 1.33333 & $4,4,2$ \\
\hline$[24,7]$ & $C_{2} \times\left(C_{3} \rtimes C_{4}\right)$ & 40 & 32 & 24 & 0.8 & 1.33333 & $4,4,2$ \\
\hline$[24,8]$ & $\left(C_{6} \times C_{2}\right) \rtimes C_{2}$ & 44 & 32 & 32 & 0.727273 & 1.33333 & $4,4,2$ \\
\hline$[24,10]$ & $C_{3} \times D_{8}$ & 36 & 24 & 24 & 0.666667 & 1 & $6,2,2$ \\
\hline$[24,11]$ & $C_{3} \times Q_{8}$ & 36 & 24 & 24 & 0.666667 & 1 & $6,2,2$ \\
\hline$[24,12]$ & & 64 & 36 & 36 & 0.5625 & 1.5 & $4,3,3$ \\
\hline$[24,13]$ & $C_{2} \times A_{4}$ & 60 & 36 & 36 & 0.6 & 1.5 & $4,3,3$ \\
\hline$[24,14]$ & $C_{2} \times C_{2} \times S_{3}$ & 40 & 32 & 32 & 0.8 & 1.33333 & $4,4,2$ \\
\hline
\end{tabular}

TABLE 1. Computational results for all nonabelian groups of order less than 25. (If $\beta$ and $\beta_{\mathrm{g}}$ differ, the corresponding values are printed bold.)

- $\beta_{\mathrm{g}}(G)$ resp. $\beta_{\mathrm{g}}$ : The TPP subgroup capacity (see definition 1.3 ).

- $\beta /|G|$ resp. $\beta_{\mathrm{g}} /|G|$ : The so called TPP ratio or TPP subgroup ratio, resp.: A parameter that measures the size of the realized problem in relation to the group size.

- $\langle n, p, m\rangle$ : The parameters of the biggest realized problem.

7.1. Computational Results for some Small Groups. Our search algorithms allow us to compute all the relevant information about TPP triples of subsets for all nonabelian groups of order less than 25. The results are shown in table 1 (computing TPP triples of subsets in groups of order greater than 24 is too time consuming at present).

7.2. Computational Results for some 2-Groups. An interesting point is, that the only groups we found with the brute-force search for subgroups that achieve $\beta / D_{3}>0.8$ are 2 -groups. The 


\begin{tabular}{|l|l|l|l|l|l|l|}
\hline GAP Id & Group & $\boldsymbol{D}_{\mathbf{3}}(\boldsymbol{G})$ & $\boldsymbol{\beta}_{\mathbf{g}}(\boldsymbol{G})$ & $\boldsymbol{\beta}_{\mathbf{g}} / \boldsymbol{D}_{\mathbf{3}}$ & $\boldsymbol{\beta}_{\mathbf{g}} /|\boldsymbol{G}|$ & $\langle\boldsymbol{n}, \boldsymbol{p}, \boldsymbol{m}\rangle$ \\
\hline \hline$[64,226]$ & $D_{8}^{2}$ & 144 & 128 & 0.888889 & 2 & $8,4,4$ \\
\hline$[128,29]$ & $\left(C_{2} \times\left(C_{8} \rtimes C_{2}\right)\right) \rtimes C_{4}$ & 304 & 256 & 0.842105 & 2 & $16,4,4$ \\
{$[128,1135]$} & $\left(C_{2}^{3} \times D_{8}\right) \rtimes C_{2}$ & 304 & 256 & 0.842105 & 2 & $16,4,4$ \\
{$[128,1142]$} & $\left(C_{2}^{3} \times D_{8}\right) \rtimes C_{2}$ & 304 & 256 & 0.842105 & 2 & $8,8,4$ \\
{$[128,1165]$} & $\left(C_{2}^{3} \times D_{8}\right) \rtimes C_{2}$ & 304 & 256 & 0.842105 & 2 & $8,8,4$ \\
{$[128,2194]$} & $C_{2} \times D_{8}^{2}$ & 288 & 256 & 0.888889 & 2 & $16,4,4$ \\
{$[128,2213]$} & $\left(C_{2} \times C_{4} \times D_{8}\right) \rtimes C_{2}$ & 288 & 256 & 0.888889 & 2 & $16,4,4$ \\
\hline
\end{tabular}

TABLE 2. Computational results for selected 2-groups.

results for groups of order 64 and 128 are shown in table 2 (The results for all nonabelian groups of order 256 are still in computation.) Details about the subgroup search can be found in subsection 7.4 .

7.3. Selected Results for $\mathrm{SL}_{n} \mathbb{F}_{q}$ and $\mathrm{PSL}_{2} \mathbb{F}_{q}$. We also tested some groups of type $\mathrm{SL}_{n} \mathbb{F}_{q}$ and $\mathrm{PSL}_{2} \mathbb{F}_{q}$. The results are shown in the tables 3 and 4 . These are groups that realize a relatively high TPP subgroup ratio $\rho(G):=\beta(G) /|G|$. No other tested groups obtain ratios bigger than 4 .

Furthermore we present a theoretical result about a lower bound for the TPP capacity of $\mathrm{SL}_{3} \mathbb{F}_{2}$ :

Lemma 7.1. The finite special linear group $\mathrm{SL}_{3} \mathbb{F}_{2}$ realizes $\langle 8,7,7\rangle$ via a TPP triple of subgroups, and its TPP capacity has the lower bound $\beta\left(\mathrm{SL}_{3} \mathbb{F}_{2}\right) \geq 392=\frac{7}{3}\left|\mathrm{SL}_{3} \mathbb{F}_{2}\right|$.

Proof. We write $G:=\mathrm{SL}_{3} \mathbb{F}_{2}$ and define a proper subset $S \subset G$ by

$$
S:=\left\{\left[\begin{array}{ccc}
1 & x & y \\
0 & 1 & z \\
0 & 0 & 1
\end{array}\right] \mid x, y, z \in \mathbb{F}_{2}\right\} .
$$

This is a subgroup of order 8, consisting of all the upper unitriangular matrices in $G$. Define two proper cyclic subgroups $T, U<G$ by

$$
T:=\left\langle t_{0}:=\left[\begin{array}{lll}
1 & 1 & 1 \\
1 & 0 & 0 \\
1 & 1 & 0
\end{array}\right]\right\rangle, U:=\left\langle u_{0}:=\left[\begin{array}{lll}
0 & 0 & 1 \\
1 & 0 & 1 \\
1 & 1 & 1
\end{array}\right]\right\rangle .
$$

The generators $t_{0}$ and $u_{0}$, as defined above, have order 7 in $G$, so $T$ and $U$ are cyclic of order 7 . We know that $T \cap U=1$ (otherwise $t_{0}$ would generate $U$, and we would have $T=U$, which is not true). In $T U:=\left\{t_{0}^{i} u_{0}^{j}: i, j \in \mathbb{Z}_{7}\right\} \subset G$ all nontrivial products $t_{0}^{i} u_{0}^{j}$, with $i, j \in \mathbb{Z}_{7}$ not both zero, are non-upper triangular matrices, and therefore $T, U, T U \subseteq(G \backslash S) \cup 1$. Therefore, we have $S \cap T U=1$. Thus $(S, T, U)$ is a (basic) TPP triple of subgroups of $G$, and by it $G$ realizes $\langle 8,7,7\rangle$ of size $8 \cdot 7 \cdot 7=392$. The TPP capacity $\beta(G)$ is at least its TPP capacity via subgroups $\beta_{\mathrm{g}}(G)$, which, by this result, is at least 392 . Since $\mathrm{SL}_{3} \mathbb{F}_{2}=\mathrm{PSL}_{3} \mathbb{F}_{2}=\mathrm{PSL}_{2} \mathbb{F}_{7}$, this result is also true for these latter groups.

Note that it follows from the tables 3 and 4 that $\beta_{\mathrm{g}}\left(\mathrm{SL}_{3} \mathbb{F}_{2}\right)=392$ holds.

7.4. Further Results. At the moment many computations are still running on three supercomputers at the universities of Jena and Halle-Wittenberg. A first goal is to compute the TPP subgroup capacity $\beta_{\mathrm{g}}$ for all nonabelian groups of order up to 1000 (except 512 and 768). We also hope that there exists an algorithm that can effectively produce basic subsets, because the computation of the TPP subset capacity $\beta$ has a complexity of $\mathcal{O}\left(8^{|G|}\right)$ without such an algorithm and it takes 8 hours to compute $\beta$ for one group of order 24 . All results (that means of the subset search for groups of order up to 24 and of the subgroup search of groups up to order 1000) can be found at http://www2.informatik.uni-halle.de/da/hedtke/tpp/. Note that some results are still missing, because they are in computation at the moment. 


\begin{tabular}{|l|r|r|r|l|l|l|}
\hline Group & $\boldsymbol{D}_{\mathbf{3}}(\boldsymbol{G})$ & $|\boldsymbol{G}|$ & $\boldsymbol{\beta}_{\mathbf{g}}(\boldsymbol{G})$ & $\boldsymbol{\beta}_{\mathbf{g}} / \boldsymbol{D}_{\mathbf{3}}$ & $\boldsymbol{\beta}_{\mathbf{g}} /|\boldsymbol{G}|$ & $\langle\boldsymbol{n}, \boldsymbol{p}, \boldsymbol{m}\rangle$ \\
\hline \hline $\mathrm{PSL}_{2} \mathbb{F}_{2}$ & 10 & 6 & 8 & 0.8 & 1.33333 & $2,2,2$ \\
$\mathrm{PSL}_{2} \mathbb{F}_{3}$ & 30 & 12 & 18 & 0.6 & 1.5 & $3,3,2$ \\
$\mathrm{PSL}_{2} \mathbb{F}_{4}$ & 244 & 60 & 108 & 0.442623 & 1.8 & $12,3,3$ \\
$\mathrm{PSL}_{2} \mathbb{F}_{5}$ & 244 & 60 & 108 & 0.442623 & 1.8 & $12,3,3$ \\
$\mathrm{PSL}_{2} \mathbb{F}_{7}$ & 1126 & 168 & 392 & 0.348135 & 2.33333 & $8,7,7$ \\
$\mathrm{PSL}_{2} \mathbb{F}_{8}$ & 4072 & 504 & 1372 & 0.336935 & 2.72222 & $14,14,7$ \\
$\mathrm{PSL}_{2} \mathbb{F}_{9}$ & 3004 & 360 & 972 & 0.323569 & 2.7 & $12,9,9$ \\
$\mathrm{PSL}_{2} \mathbb{F}_{11}$ & 7038 & 660 & 1980 & 0.28133 & 3 & $55,6,6$ \\
PSL $_{2} \mathbb{F}_{13}$ & 13556 & 1092 & 3276 & 0.241664 & 3 & $78,7,6$ \\
PSL $_{2} \mathbb{F}_{17}$ & 40252 & 2448 & 10368 & 0.257577 & 4.23529 & $24,24,18$ \\
PSL $_{2} \mathbb{F}_{19}$ & 63646 & 3420 & 14400 & 0.226251 & 4.21053 & $60,20,12$ \\
\hline
\end{tabular}

TABLE 3. Computational results for selected groups of type $\mathrm{PSL}_{2} \mathbb{F}_{q}$.

\begin{tabular}{l|l|r|r|r|l|l|l|}
\hline Group & $\boldsymbol{D}_{\mathbf{3}}(\boldsymbol{G})$ & $|\boldsymbol{G}|$ & $\boldsymbol{\beta}_{\mathrm{g}}(\boldsymbol{G})$ & $\boldsymbol{\beta}_{\mathrm{g}} / \boldsymbol{D}_{\mathbf{3}}$ & $\boldsymbol{\beta}_{\mathrm{g}} /|\boldsymbol{G}|$ & $\langle\boldsymbol{n}, \boldsymbol{p}, \boldsymbol{m}\rangle$ \\
\hline \hline $\mathrm{SL}_{2} \mathbb{F}_{2}$ & 10 & 6 & 8 & 0.8 & 1.33333 & $2,2,2$ \\
$\mathrm{SL}_{2} \mathbb{F}_{3}$ & 54 & 24 & 36 & 0.666667 & 1.5 & $4,3,3$ \\
$\mathrm{SL}_{2} \mathbb{F}_{4}$ & 244 & 60 & 108 & 0.442623 & 1.8 & $12,3,3$ \\
$\mathrm{SL}_{2} \mathbb{F}_{5}$ & 540 & 120 & 216 & 0.4 & 1.8 & $24,3,3$ \\
$\mathrm{SL}_{2} \mathbb{F}_{7}$ & 2198 & 336 & 784 & 0.356688 & 2.33333 & $16,7,7$ \\
$\mathrm{SL}_{2} \mathbb{F}_{8}$ & 4072 & 504 & 1372 & 0.336935 & 2.72222 & $14,14,7$ \\
\hline $\mathrm{SL}_{3} \mathbb{F}_{2}$ & 1126 & 168 & 392 & 0.348135 & 2.33333 & $8,7,7$ \\
\hline
\end{tabular}

\section{ACKNOWLEDGEMENTS}

We would like to thank David J. Green, Peter M. Neumann, Luke Oeding and Maxim PETRUnin for their helpful comments and suggestions. We also thank the Friedrich-SchillerUniversity Jena and the Martin-Luther-University Halle-Wittenberg which allowed us to use their supercomputers.

\section{REFERENCES}

[Aichinger et. al 2008] E. Aichinger, F. Binder, J. Ecker, P. MAYr and C. NÖBAuer, SONATA - system of nearrings and their applications, GAP package, Version 2.4, 2008. http://www.algebra.uni-linz.ac.at/Sonata/

[D'Alberto et al. 2009] P. D'Alberto and A. Nicolau, 'Adaptive Winograd's Matrix Multiplications', ACM Trans. Math. Softw. 36, 1, Article 3, 2009.

[Alperin et al. 1991] J. L. Alperin and R. Bell, Groups and Representations, Springer, 1991.

[Bürgisser et. al 1997] P. Bürgisser, M. Clausen and M. A. Shokrollahi, Algebraic Complexity Theory, Springer, Grundlehren der mathematischen Wissenschaften, Vol. 315, ISBN 978-3-540-60582-9, 1997.

[Burnett 2010] E. BuRnetT, 'Indexing and enumerating subsets of a given size', Blog The Lowly Programmer, Entry from April 2010, http://www.thelowlyprogrammer.com/2010/04/indexing-and-enumerating-subsets-of .html.

[Cohn et al. 2003] H. Cohn and C. Umans, 'A Group-theoretic Approach to Fast Matrix Multiplication', Proceedings of the $44^{\text {th }}$ Annual Symposium on Foundations of Computer Science, 11-14 October 2003, Cambridge, MA, IEEE Computer Society, pp. 438-449.

[Cohn et al. 2005] H. Cohn, R. Kleinberg, B. Szegedy and C. Umans, 'Group-theoretic Algorithms for Matrix Multiplication', Proceedings of the $46^{\text {th }}$ Annual Symposium on Foundations of Computer Science, 23-25 October 2005, Pittsburgh, PA, IEEE Computer Society, pp. 379-388.

[Coppersmith et al. 1987] D. Coppersmith and S. Winograd, 'Matrix Multiplication via Arithmetic Progressions', STOC '87: Proc. of the nineteenth annual ACM symposium on Theory of Computing.

[GAP 2008] The GAP Group: GAP - Groups, Algorithms, and Programming, Version 4.4.12, 2008. http://www.gap-system.org

[Hedtke 2011] I. Hedtke, 'A Note on the Group-theoretic Approach to Fast Matrix Multiplication', ArXiv eprint, http://arxiv.org/abs/1101.5598, 2011.

[James et al. 2011] G. JAMES and M. LIEBECK, Representations and Characters of Groups, Cambridge University Press, $2^{\text {nd }}$ edition, 2001. 
[Neumann 2011] P. M. Neumann, 'A note on the triple product property for subsets of finite groups', to appear in Journal of Computation and Mathematics, London Mathematical Society, 2011.

[Neumann 1994] P. M. Neumann, Groups and Geometry, Oxford Science Publications, 1994.

[Orem 2009] H. Orem, 'Fast Matrix Multiplication via Group Actions', Undergraduate thesis, Departement of Mathematics, Harvey Mudd College, 2009. http://www.ma.utexas.edu/users/horem/

[Strassen 1969] V. Strassen, 'Gaussian Elimination is not Optimal', Numer. Math. 13:354-356, 1969.

Mathematical Institute, University of Jena, D-07737 Jena, Germany

E-mail address: Ivo.Hedtke@uni-jena.de

29 Stephen Road, Oxford OX3 9Ay, England

E-mail address: sandeepr.murthy@gmail.com 